\title{
Genetic and Environmental Factors Contributing to Visceral Adiposity in Asian Populations
}

\author{
Rachel Williams, Muthu Periasamy \\ Department of Internal Medicine, University of Central Florida (UCF) College of Medicine, Burnett School of Biomedical \\ Sciences, Orlando, FL, USA
}

Obesity-associated metabolic illnesses are increasing at an alarming rate in Asian countries. A common feature observed in the Asian population is a higher incidence of abdominal obesity - the "skinny-fat" Asian syndrome. In this review, we critically evaluate the relative roles of genetics and environmental factors on fat distribution in Asian populations. While there is an upward trend in obesity among most Asian countries, it appears particularly conspicuous in Malaysia. We propose a novel theory, the Malaysian gene-environment multiplier hypothesis, which explains how ancestral variations in feast-and-famine cycles contribute to inherited genetic predispositions that, when acted on by modern-day stressors - most notably, urbanization, westernization, lifestyle changes, dietary transitions, cultural pressures, and stress - contribute to increased visceral adiposity in Asian populations. At present, the major determinants contributing to visceral adiposity in Asians are far from conclusive, but we seek to highlight critical areas for further research.

Keywords: Intra-abdominal fat; Adiposity; Asian

\section{INTRODUCTION}

Obesity results from an imbalance between energy intake and expenditure over an extended period. It is generally characterized as excessive fat storage and an increase in the ratio of fat to muscle. Obesity has become a global epidemic during the last decade and is considered a major causative factor for numerous illnesses and increased morbidity [1]. This gives rise to the question "why store fat at all"? All vertebrates store fat in their bodies. Fat is essential for the survival of an organism and can sustain life during starvation and famine. It is a major component of the living cell structure, as most organelles are composed of lipid membranes (nuclear, Golgi, and endoplasmic reticulum membranes). Fat is a major energy substrate due to its high caloric content and it is essential for generation of adenosine triphosphate. The body has evolved a mechanism to store fat; when intake of glucose and fat exceeds energy demand, it is consequently stored in different locations [2]. Presently, it is unclear whether there are any advantages to storing fat in particular locations in the body. It is, however, interesting that fat storage and distribution varies between different ethnicities and to some extent between individuals. There is also scientific evidence suggesting that subcutaneous fat is healthier than visceral fat, as increased visceral fat is associated with insulin resistance and lipotoxicity [3].

Fat distribution patterns vary among different racial and ethnic groups and reflect racial and ethnic differences in anthropometrics. Generally, Hispanic and African-American women
Received: 19 July 2020, Revised: 1 October 2020, Accepted: 12 December 2020 Corresponding author: Muthu Periasamy

Department of Internal Medicine, University of Central Florida (UCF) College of Medicine, Burnett School of Biomedical Sciences (Room 541), 6900 Lake Nona Blvd, Orlando, FL 32827, USA

Tel: +1-407-266-7049, Fax: +1-407-266-2689,

E-mail: muthu.periasamy@ucf.edu

\section{Copyright $(2020$ Korean Endocrine Society}

This is an Open Access article distributed under the terms of the Creative Commons Attribution Non-Commercial License (https://creativecommons.org/ licenses/by-nc/4.0/) which permits unrestricted non-commercial use, distribution, and reproduction in any medium, provided the original work is properly cited. 
tend to store fat around the hips, buttocks, and thighs, a physiological phenomenon characteristic of the gynoid ("pear shape") body, while Asian women tend to store fat around the abdomen, characteristic of the android or "apple shape" body [4]. Significant differences in abdominal fat storage have been observed across racial and ethnic analyses, although the differences are less marked in men than women [5,6]. Increasingly, recent studies have indicated the prevalence of higher abdominal fat in Asian populations [7-13].

This finding emphasizes the relationship between not only excess fat storage in general, but the location of fat storage, as Asians demonstrate greater visceral fat storage despite lower body mass index (BMI) values. Consequently, higher levels of visceral fat have been consistently linked to higher risk of metabolic diseases such as type 2 diabetes, hyperglycemia, and higher triglyceride levels, as well as cardiovascular diseases and hypertension [14,15]. Given this discrepancy, recommendations have been made that incorporate waist circumference and other anthropomorphic measurements in addition to BMI in predicting metabolic risk [16]. This is accompanied by race-specific modifications to BMI and associated benchmarks.

Asia is home to nearly 4.7 billion people, which amounts to approximately $61 \%$ of the global population. It represents some of the largest economies in the 21 st century and continues to grow at a fast pace, shifting people from rural to urban lifestyles. This rapid economic growth has created a rich middle class and affluent lifestyle among many Asians. With increased economic growth and affluence, there has been a shift from traditional to Western lifestyles, often accompanied by the replacement of traditional cuisine for westernized diets. With an increase in economic growth, Asia has also seen a steady increase in obesity and diabetes during the last three decades. China and India have 116 and 77 million people living with type 2 diabetes, respectively, and India is expected to surpass China by 2050 if this problem is not controlled [17].

The Asian biological propensity to store fat predominantly in the abdominal region poses an important question: Why do Asians have a genetic predisposition to store fat viscerally, and to what extent do genetics and the environment play a role in modifying fat distribution?

\section{GENETIC FOUNDATION}

\section{An illustrative theory: the Yajnik-Yudkin hypothesis (Y-Y paradox)}

The tendency toward visceral fat storage in Asians is illustrated by the Yudkin-Yajnik paradox. Yudkin, a British doctor, and his colleague Yajnik, a South Asian doctor, demonstrated significant differences in fat composition despite having similar BMIs. Though Yajnik appeared physically slenderer, body scans showed a higher fat-content-to-body-weight ratio. This "thinfat" anomaly illustrates a trend in which Asians with comparable (if not lesser) BMI measurements as Caucasians have higher body fat content, particularly visceral and abdominal fat, as well as lower skeletal muscle mass and higher rates of insulin resistance [18].

\section{Shared metabolic-related genes}

Recent studies have identified genes involved in metabolic functioning that may influence fat distribution, body structure, and fat metabolism pathways integral to fat storage and utilization. Genetic similarities and shared genomic lineages between individuals of the same ethnicity may be responsible for patterns of metabolic syndromes and anthropomorphic features characteristic of certain ethnicities.

Genome-wide association studies (GWAS) have been conducted on the heritability of fat distribution. A study by Lotta et al. [19] identified 202 independent genetic variants associated with higher BMI-adjusted waist-to-hip-ratio (WHR) and unadjusted WHR that were also associated with higher blood pressure, triglyceride levels, and risk of diabetes. Certain genetic variants are known to influence patterns of compartmental fat distribution. A meta-analysis by Heid et al. [20] indicated 13 loci associated with WHR and revealed sexual dimorphism in the genetic basis of fat distribution. Accordingly, sets of genetic loci have been identified and linked with roles in body fat regulation, insulin sensitivity, transcriptional regulation of metabolically active tissues, and enriched expression in adipocyte-related tissues [21].

Various loci have been identified whose role in regulating specific metabolic functions is to collectively influence the phenotypic expression of fat distribution. For instance, neuropeptide Y (NPY) has been shown to have adipogenic effects on energy storage, food intake, and obesity, as well as cardiovascular regulation. A study of the single-nucleotide polymorphism in the rs 16147 allele of the NPY gene revealed differences in waist circumference reduction and adiposity between the $\mathrm{C}$ and $\mathrm{T}$ allele variants when participants were fed a caloric-deficient, but high-fat diet: those with the $\mathrm{T}$ allele showed smaller reduction in waist circumference compared to those with the $\mathrm{C}$ allele, along with more adverse changes in abdominal fat content compared to those fed a low-fat diet and a greater propensity to regain ab- 
dominal fat 24 months into the dietary intervention. Significant differences in waist circumference, total adipose tissue, visceral adipose tissue (VAT), and subcutaneous adipose tissue between those fed a high-fat and low-fat diet were also observed [22].

Interestingly, African-American men and women demonstrate the largest relative musculoskeletal mass, as well as the lowest mean trunk, visceral, and liver adiposity compared to Caucasians, Asians, and Hispanics, further illustrating consistent patterns of inheritance in body composition and metabolic function perpetuated by shared genomic histories $[23,24]$.

\section{THE ROLE OF SEX IN FAT DISTRIBUTION}

Sex differences in fat distribution are well established (sexual dimorphism), but ethnic differences in the location of fat storage between sexes requires further exploration [18]. Women typically store fat inferiorly (lower body) while men tend to store fat superiorly (upper body). Differences in hormone levels are predominantly responsible (physiological actions of estrogen vs. testosterone) for the marked difference in location of fat deposition between males and females.

Certain fat distribution-related traits appear significant in heritability between males and females (WHR, WHR adjusted for BMI, waist circumference, hip circumference, etc.). Sexually dimorphic loci associated with anthropometric factors and fat distribution have been implicated by GWAS [25]. Genetic loci linked to hip circumference, for example, exhibit larger effects in women, while loci linked to waist circumference appear more active in men [21]. Men appear to store more fat viscerally, while women have higher levels of subcutaneous fat $[5,25]$.

Female fat distribution patterns differ considerably from those in males - as to facilitate reproduction and fetal nourishmentand as such, women demonstrate greater variability in fat distribution across different ethnicities. Nevertheless, both Asian men and women demonstrate consistently higher levels of visceral adiposity compared to other ethnicities.

Various studies have found a higher percentage of abdominal visceral fat in women of Asian heritage than in Caucasian women. Asian women appear to store more fat abdominally and viscerally than Caucasian women of comparable adiposity. An examination of percent liver fat and fatty liver also showed higher levels in Japanese-American women than in Caucasian women [7]. Similar findings, on a less pronounced scale, are reflected in Asian men [24]. Japanese men, as with Japanese women, demonstrated higher levels of VAT, particularly liver fat, compared to Caucasian men [26].

\section{ETHNIC DIFFERENCES AMONG ASIANS}

It has been well established that Asians store a higher percentage of body fat abdominally compared other races. However, it is worth noting ethnic differences in fat composition and distribution within the Asian population. Within the United States, Asian-Americans represent over 20 different national origins [27]; significant physiological, cultural, and socioeconomic differences between Asian ethnicities should be noted when analyzing fat distribution for the Asian population as a whole.

Among Asian Indians, Malays, and Chinese, Asian Indians presented with the highest percent body fat and Chinese with the lowest (Fig. 1). Overall, East Asians exhibit lower percent body fat than South and Southeast Asians [13]. Among AsianAmericans, ethnic groups with lower socioeconomic status (Vietnamese, Hmong, Cambodian) were associated with higher risk of being overweight [27].

\section{FAT DISTRIBUTION IN ASIAN CHILDREN}

Abdominal fat distribution is significantly greater in Asian children than in children of different ethnicities. After controlling for BMI, both Asian children and adolescents exhibited higher waist circumference and greater visceral fat deposits than Caucasian children of the same age group, reflecting later patterns of increased visceral fat deposits in Asian men and women compared to Caucasian men and women $[8,13]$. However, it is difficult to separate significant ethnic differences in fat distribution from confounding variables such as normal hormonal and physiological changes during growth, development, and puberty when examining children and adolescents.

Differences in fat distribution have been found in Asian prepubertal children from different origins. For instance, Chinese and Thai children were found to have a higher waist circumference as well as higher trunk fat deposits than their Lebanese and Malay counterparts [8]. Differences in fat composition exist within Asian regions of similar climate and environment, as well as between the sexes, a phenomenon that may illustrate the genetic influence of fat distribution: Thai girls displayed higher percentage body fat than Malay and Filipino girls; Filipino boys displayed lower percentage body fat than Malay and Thai boys [13].

Differences in fat distribution present at birth may further reinforce the role of genetics in the biological regulation of fat storage as opposed to environmental factors. Asian, African-American, and Hispanic newborns exhibit greater central adipose deposition and percentage fat mass than Caucasian newborns [28]. 
Percentage of Indians overweight or obese, 1975-2015

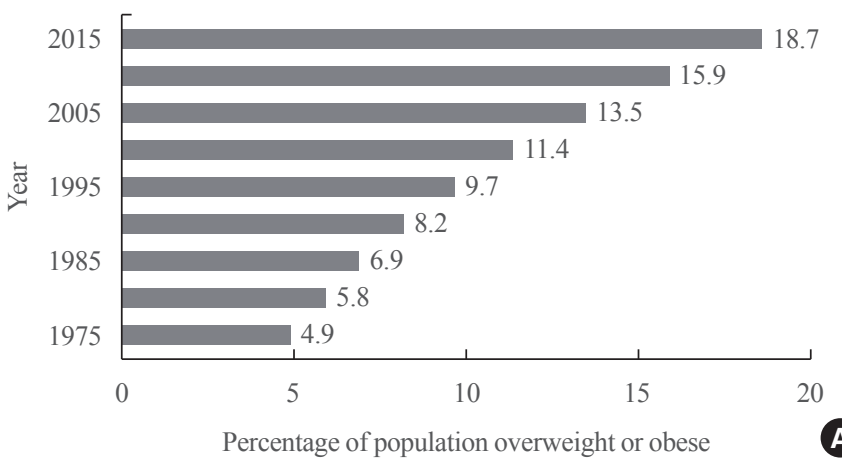

Percentage of Koreans overweight or obese, 1975-2015

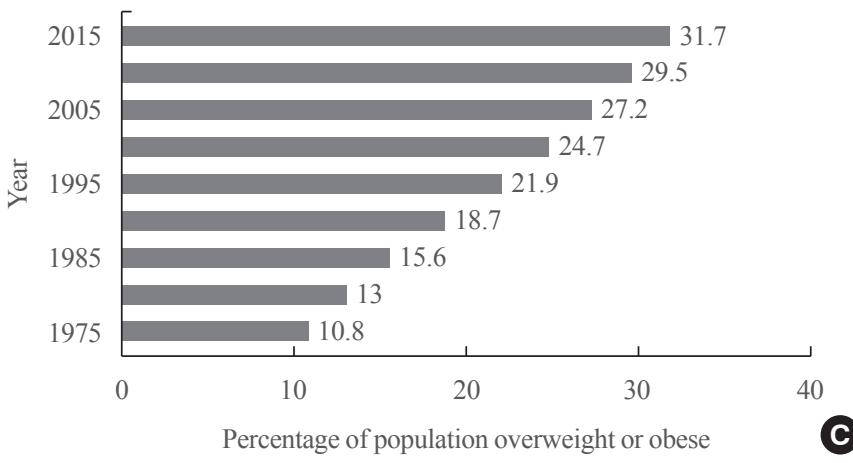

Percentage of Vietnamese overweight or obese, 1975-2015

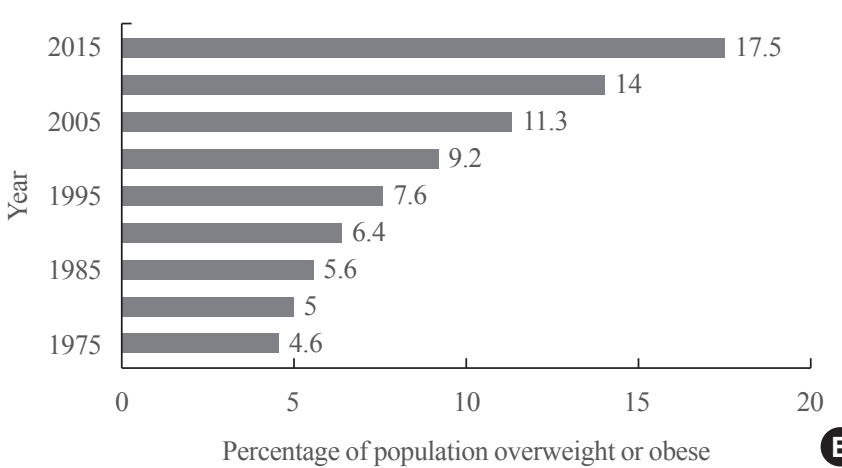

Percentage of Chinese overweight or obese, 1975-2015

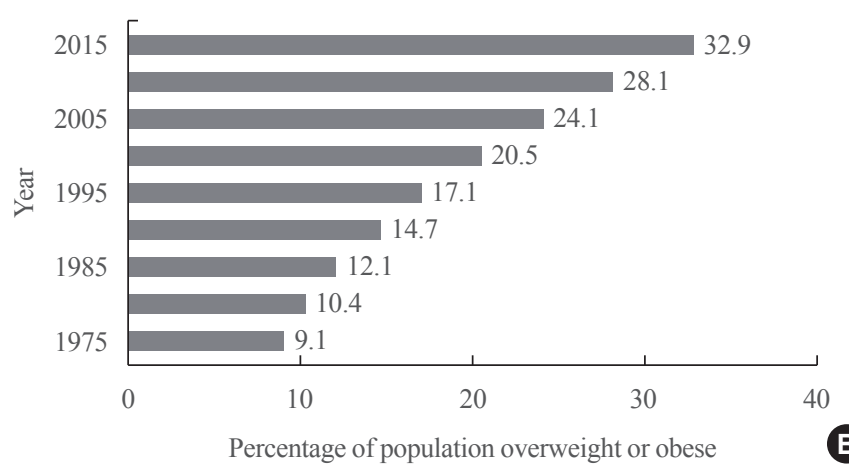

Percentage of Japanese overweight or obese, 1975-2015

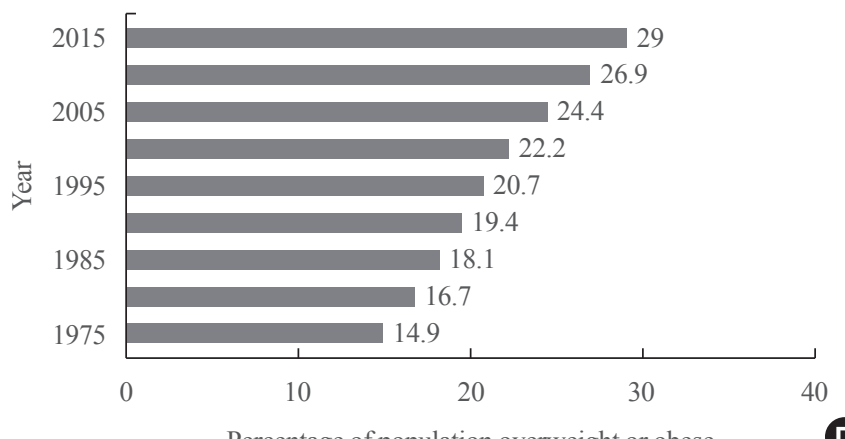

Percentage of Malaysians overweight or obese, 1975-2015

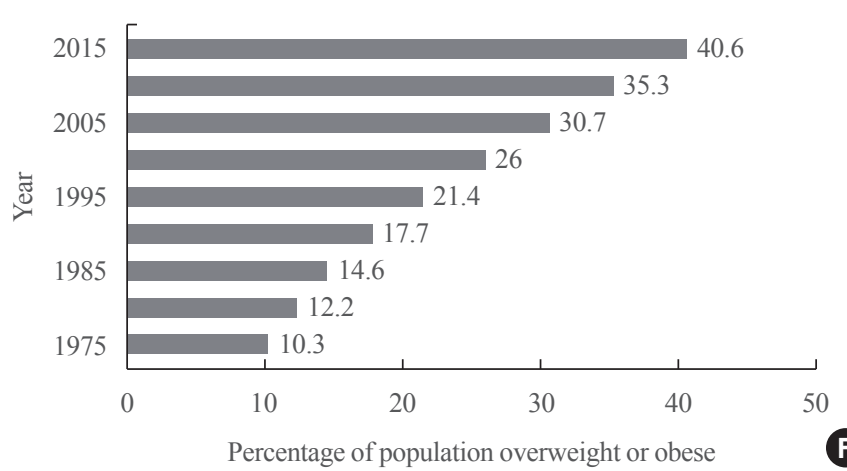

Fig. 1. Percentage of the overweight or obese population for select South and Southeast Asian countries: (A) Indians, (B) Chinese, (C) Koreans, (D) Japanese, (E) Vietnamese, and (F) Malaysians. Overweight is defined as having a body mass index (BMI) greater than or equal to $25 \mathrm{~kg} / \mathrm{m}^{2}$. Obesity is defined as having a BMI greater than or equal to $30 \mathrm{~kg} / \mathrm{m}^{2}$. BMI is an individual's weight (in kilograms) divided by height (in meters) squared. Graphs were derived from data from Our World in Data "https://ourworldindata.org/obesity" obesity by "https:// ourworldindata.org/team" Hannah Ritchie and Max Roser, used under “https://creativecommons.org/licenses/by/4.0/” CC BY 4.0 [73].

In comparing United Kingdom-born South Asian infants and United Kingdom-born Caucasian infants, infants of South Asian descent presented with greater fat mass than Caucasian infants, as well as greater subscapular skin-fold thickness [28].

Ethnic differences in fat distribution follow a similar pattern into childhood and adulthood. In a study of British schoolchil- dren aged 5 to 7, South Asian girls and boys exhibited a higher body fat percentage than white and African-Caribbean ethnic groups. Other studies have found less extremity fat in both Asian girls and boys, but higher levels of truncal fat in Asian girls compared to Caucasian girls [29]. Interestingly, a study by Freedman et al. [30] found that thin Asian children tended to 
have higher percent body fat when compared to Caucasian children of similar BMI, while overweight Asian children exhibited lower percent body fat compared to Caucasian children. Consistent with previous studies, the study found that normal-weight Asian children displayed higher levels of body fat than Caucasian children [30]. Continuing into adolescence, South Asian females and males demonstrated a higher body fat percentage than Caucasian and African-Caribbean adolescents [31].

Childhood and adolescent studies demonstrate consistent ethnic differences in waist circumference and abdominal fat deposition. Differences are more pronounced in females than males, with females generally having a greater difference in fat mass than males. Differences in fat distribution present in adulthood appear to persist into old age.

\section{ENVIRONMENTAL IMPACT}

The environmental impact on fat distribution cannot be understated, and it is one not specific to Asian populations, but applicable worldwide. Increasingly, fast-paced technology environments, capitalistic ideals, and the globalization of Western culture have led to diets drastically different from that of Homo sapiens predecessors. There has been an increased homogenization of diets around the world, such that meals in developed and developing countries tend toward being calorie-dense, nutrientrich, and highly processed regardless of the country. Humans have adopted more sedentary lifestyles in addition to altered diets. Obesity on a global scale has nearly tripled from 1975 to 2016, with an alarming increase in childhood obesity [2]. This breaks down to a simple metabolic consequence: excess, unused calories are stored in the body as fat, and regardless of where the excess fat is stored, it must be stored somewhere. An Asian individual may initially store more excess fat around their abdomen, while a Caucasian individual may initially store excess fat predominantly in their appendages, but if both individuals exceed the fat storage capacity for that portion of their body, the fat must be stored elsewhere, eventually leading to fat stored in all places, regardless of genetic heritage. Through this, we see an increasing global homogenization of body shape more strongly influenced by the environment than genetics.

Several studies have directly cited environmental factors as a key influence in body fat distribution. A comparison between groups of identical race, but different environments (for instance, Japanese in Japan vs. Japanese in America) showed significant difference in percentage of VAT (VAT\%); a comparison between two different races, but identical environments (Cauca- sians vs. Japanese-Americans) showed no significant difference in $\mathrm{VAT} \%$. VAT\% remained larger in Chinese, Japanese, and Filipino men than in Caucasian men of the same level of obesity [9].

\section{DIET}

Diet is perhaps one of the most immediate factors influencing fat distribution. In general, diets worldwide have become more processed and less plant-based, resulting in increased obesogenic nutritional content such as higher levels of sugar and fat [32]. This is especially apparent in the diets of developing Asian countries, where globalization trends, internal modernization, and the growing appeal of Western-style fast food have led to increasingly westernized diets $[32,33]$. Dietary shifts from coarse grains to refined rice and wheat often accompany rapid economic development [34]. Moreover, second-generation Asian immigrants are more likely to diverge from their native country's diet. These diets, as in the illustrative case of Japanese-Brazilian 'Nissei' (the children of first-generation Japanese immigrants) tend to follow Western trends and include a higher percentage of sugar and fat compared to traditional Japanese diets [35].

More specific to the Asian population is white rice consumption. White rice contains a higher glycemic index than brown rice and other carbohydrates (bread, pasta, etc.) and is metabolized more quickly than brown rice [36]. Compared to brown rice, white rice exhibits higher postprandial levels of glucose and insulin; long-term consumption may stimulate insulin resistance and risk of type 2 diabetes, along with increased risk of associated metabolic syndromes [36,37]. Metabolic responses following white rice consumption may be responsible for increased abdominal adiposity, although the specific mechanism is not yet known. White rice is deeply embedded in Asian culture, history, and heritage and remains a staple food crop today. The rice consumption of Asians is markedly greater than that of Caucasians: three to four servings a day compared with one to two servings a week [35]. Thus, the centrality of white rice to Asian cuisine presents another potential contributing factor to increased abdominal fat deposits.

\section{CHANGING LIFESTYLE AND STRESS}

There has been an enduring shift from predominantly rural living to an urban lifestyle, especially in countries like India and China, where better-paying jobs have motivated increased urbanization (Fig. 2). This has created an exodus of rural workers 


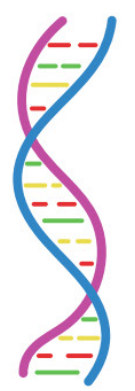

Genetic Foundation

Genetic propensity for storing fat viscerally, shared genes involved in metabolic processes and fat deposition

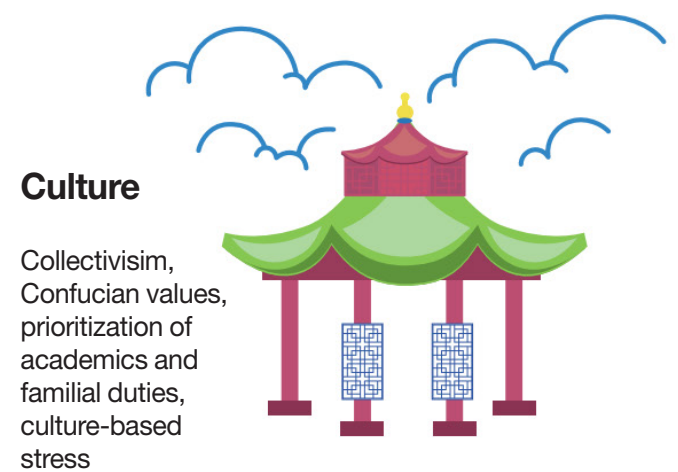

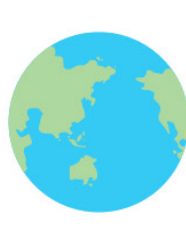

\section{Evolutionary History}

Thrifty gene hypothesis, feast and famine cycles

\section{Lifestyle \\ Urbanization, sedentary occupations, stress}

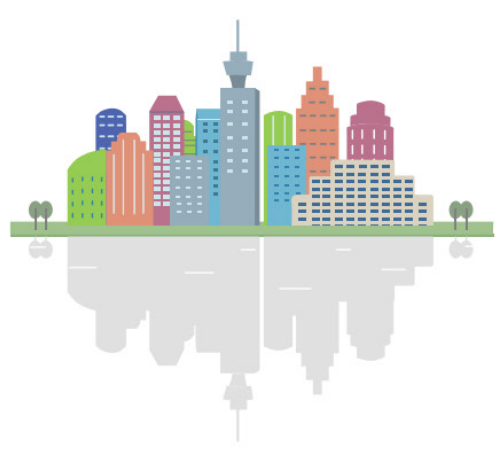

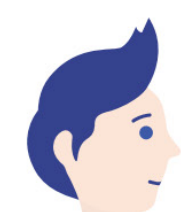

\section{Exercise}

Less physical activity, sedentary activities

Subcutaneous fat

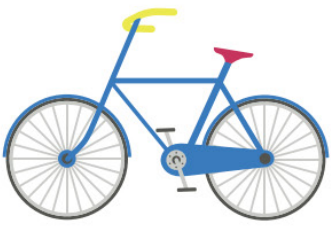

Visceral fat

\section{Diet}

Increased incorporation of sugary, processed foods, foods with high glycemic index (rice, noodles)
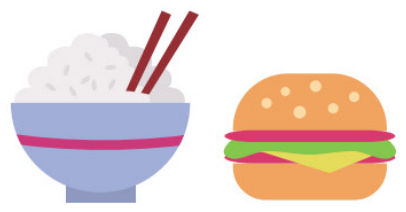

Fig. 2. The complex interplay between genetic and environmental factors contributing to increased visceral adiposity in Asian populations. Many factors contribute to the Asian propensity to store fat viscerally. Of these, we propose the Malaysian gene-environment multiplier hypothesis in which a population's genetic foundation — developed over thousands of years and varying according to regional feast-and-famine cycles - is increasingly modified when an individual is exposed to modern-day conditions such as altered diets, urbanization, and reduced physical activity.

into the urban climate and crowded living in poor sanitary conditions. Industrial jobs are often associated with long hours, night shifts, and sleep deprivation. In developed Asian countries such as Korea, upward trends in abdominal obesity may be explained by an increasingly strong exposure to and adoption of
Western lifestyle and cuisine [38]. An increasing preference for Western diets may result in the substitution of healthier traditional cuisine with Western food options that are particularly high in added sugar, saturated fats, and preservatives [39].

In addition, Asians tend to live in clusters, often with extend- 
ed family in the same household. Such conditions may also increase stress in terms of dealing with family members (in-laws, the difficulties of being in the "sandwich generation" where one is essentially "sandwiched" between responsibilities in taking care of one's elderly parents while also raising one's own children, etc.) and contribute to stress-mediated abnormal fat metabolism. Cortisol, a hormone released during times of stress, has been shown to increase visceral fat deposition [40]. Prolonged, chronic stress may lead to hyperactivity of the hypothalamic-pituitary-adrenal axis, which is invoked in cortisol-mediated visceral fat accumulation [41]. Visceral adiposity itself appears more responsive to provoking and sustaining adverse hormonal cascades (cortisol, leptin resistance, catecholamines) and proinflammatory factors (interleukin-6), promoting a vicious cycle of continued visceral adipose accumulation [42]. One should then ask whether the pressures imposed by Asian collectivistic cultures and cultural standards are contributing to increased, prolonged stress and consequent morphological and metabolic changes in the Asian population.

Increased visceral adiposity may function as an evolutionary souvenir exacerbated by present-day stressors. In times where Homo sapiens faced short-term stresses governed by fight-orflight, cortisol activity and altered metabolic function may have facilitated greater reproductive and survivorship advantage. However, chronic stress in technologically advanced societies presents modern-day metabolic disadvantages, primarily in the form of increased visceral adipose deposits, altered fat metabolism, and associated risks of cardiovascular disease, diabetes, and metabolic syndrome.

\section{CULTURAL DIFFERENCES AND THE ROLE OF EXERCISE IN FAT DISTRIBUTION}

Exercise is known to decrease visceral fat deposits, with highintensity exercise training demonstrating greater and faster abdominal and subcutaneous fat loss $[29,43,44]$. Regular aerobic and resistance exercise prevents visceral fat gain and discourages fat regain following weight loss [45]. Even low-to-moderate amounts of exercise exert preventative effects on visceral fat gain [14]. Accordingly, increases in visceral abdominal fat have been observed in sedentary individuals [14].

Regular exercise provides significant reduction in stress. Following exposure to laboratory stressors, physically trained men demonstrated lower cortisol and heart rate stress responses, as well as increased calmness, better mood, and lower anxiety levels than physically untrained men [46]. Reduced mortality was observed in patients with coronary artery disease who engaged in exercise training, a potential mechanism being exercise-mediated reduction in psychosocial stress [47].

The benefits of exercise extend to improved mood, stress, and anxiety, as well as reduced inflammation, improved cognitive function, and alteration of physiological mechanisms to facilitate better overall health $[48,49]$. As stress has been shown to influence abdominal adipose deposition patterns, exercise may both directly and indirectly help to prevent and decrease potential and existing visceral fat.

As such, lifestyle choices play a major role in body composition and anthropometry. Compared to Caucasians and AfricanAmericans, Asians generally display lower levels of physical activity and are less likely to meet daily-recommended requirements for physical exercise [50-59]. Reduced physical activity in the Asian population may contribute to greater abdominal fat deposition. Among a study of children in the United Kingdom, South Asian children were found to have lower mean activity levels and higher periods of sedentary activity than Caucasians and African-Caribbeans, both factors associated with increased risk of cardiovascular disease and type 2 diabetes later in life [51]. Asian girls participated in less physical exercise than Asian boys $[51,55]$. Cultural differences may be responsible for disparities in mean exercise levels. Asian families are more likely to prioritize academics over athletic pursuits, especially in Confucian-based cultures where success is associated with educational attainment and economic standing. This is further magnified by Western capitalistic ideals that associate success with monetary measurements $[59,60]$. As such, Asian children show increased participation in sedentary activities compared to other ethnicities: studying, computer use, homework, after-school academies, etc. [59].

Second-generation Asian-Americans were more likely to engage in low-to-moderate forms of physical activity than foreignborn and first-generation Asian-Americans, although levels of physical activity remained lower than in other ethnicities $[52,55]$. Possible reasons for increased physical activity in the younger, American-born Asian generation as compared to Asian immigrants include a more positive outlook toward physical activity, as well as increased exposure to Western norms and practices [55]. Engagement in regular physical activity appears to be positively correlated with residence time in the United States: immigrants with longer residence times reported higher physical activity levels compared to immigrants with less residence time $[52,55,61]$. This may be due to increased exposure to Western ideals and acculturation to Western habits [61]. For instance, the 
common collectivistic culture of Asian populations prioritizes the group over the individual, thereby putting less priority on recreational or personal pursuits (such as leisure-time physical activity) in lieu of occupation, domestic work, and family matters. Common reported barriers to physical activity for more recently immigrated Asian families include cost, family commitments, lack of free time, language barriers, and safety concerns [58]. Migration to the United States and the resultant acculturation of Western individualistic practices (with more emphasis on personal health and wellbeing) may be responsible for increased physical activity level in longer-staying immigrants and their children.

Among female immigrants, exercise appeared to hold a negative stigma, often labeled as a "selfish act" and one lesser prioritized compared to domestic duties and caretaking [54]. Mothers in Hong Kong were more likely to obtain incidental physical activity from household chores than leisure-time exercise; however, recent increases in hiring domestic help and consequent redistribution of housework leaves less opportunity for daily physical activity [60].

Returning to the earlier Yudkin-Yajnik hypothesis, it is worth noting that though Yudkin and Yajnik were of comparable BMIs, Yudkin participated in regular physical activity, while Yajnik maintained a relatively sedentary lifestyle [18]. Given the extensive benefits of exercise, the tendency toward lower physical activity levels and increased sedentary activity in Asian populations may help to explain existing abdominal visceral fat distribution patterns.

\section{THE COMPLEX INTERPLAY BETWEEN GENETICS AND THE ENVIRONMENT}

Much of Asian anthropometry and fat distribution cannot be isolated to a single factor, but a complex interplay between genetics and the environment (Fig. 2). First proposed in 1962, the thrifty gene hypothesis demonstrates how selective pressures during periods of famine and scarcity favor "thrifty genes" with circumstantially advantageous features - more efficient fat storage, slower fat metabolism, etc. - that increase chances of survival when faced with nutritional insufficiencies. This, however, becomes counterproductive and metabolically disadvantageous in times of overabundance, such as when transitioning from poverty to affluence, from labor-intensive work to sedentary lifestyles, or from traditional diets to westernized, processed meals [62]. More recent reevaluations of the hypothesis have led to modified versions such as the thrifty phenotype hypothe- sis (fetal and infant metabolic adjustments considered "nutritionally thrifty" are made in response to adverse early environments, such as famine and nutritional shortage), the thrifty genotype hypothesis (genes such as those that increase fat storage are not, in fact, selected for, but persist in the population due to genetic drift), and the thrifty epigenetic hypothesis (the acquisition of metabolic thrift occurs in the early developmental period when exposed to nutritionally disadvantageous environments; exposure alters expression of genetic factors and can be passed down and subsequently altered according to environmental conditions) [34,63-65].

Evidence for epigenetic changes in metabolism as a potential contributor to altered fat distribution are especially evident when considering the drastic shift in diet and lifestyle patterns over the past century. Indeed, the sudden rise in obesity and type 2 diabetes can most likely be attributed to environmental factors and possible epigenetic changes, as the span of even a couple centuries would be too short for an adaptive evolutionary mechanism to develop within the population. The direct transcription and translation of DNA and subsequent expression of the gene product has been well established as a non-linear process; posttranslational modifications such as DNA methylation, histone modification, and phosphorylation provide additional avenues for regulation of gene expression [66]. Notable environmental features supporting epigenetic changes in fat distribution include early developmental exposure to adverse environments (poor nutritional intrauterine environment, famine exposure in early-life, poor childhood nutrition) which later present detrimental effects when exposed to nutrient-rich, sedentary environments, rapid modernization and economic development, significant lifestyle changes due to urbanization, and migration from underdeveloped to developed countries [34].

The role of epigenetics in influencing fat distribution has been emphasized in studies analyzing fetal and infant exposure to nutritionally adverse intrauterine and early-life conditions. In a study following fetuses, infants, and young children during the Chinese famine of 1961, fetuses and infants born to relatively malnourished mothers were later shown to have higher rates of hyperglycemia later in adulthood [67]. Similar results were reported in the 1944 to 1945 Dutch famine studies, where famineexposed infants and children exhibited decreased glucose tolerance as a potential consequence of dysfunctional insulin secretion [68]. Periods of nutritional scarcity may trigger an altered metabolic response to enable the fetus to survive using less food with less energy expenditure - essentially "holding on" to the scarce nutrients it is supplied with. Epigenetic changes for genes 
suspected to regulate growth and metabolism have been observed in famine-exposed children [69]. Overall, these results suggest that early-life exposure to nutritionally adverse environments stimulate metabolic changes (more efficient fat storage, altered insulin secretion, etc.) that negatively manifest when children of famine transition to affluent lifestyles later in life. Such metabolic and epigenetic changes may be responsible for ethnic differences in fat distribution patterns as a result of cumulative transgenerational inheritance of specific famine-induced epigenetic changes from parents to offspring in famineexposed populations [70].

Contemporary fat distribution patterns can be attributed to variations in ancestral environmental adaptations, namely feastand-famine cycles. In reference to the above famine exposure studies, cycles of feast-and-famine throughout history have been more frequent in the Asian continent than in the European continent. Frequent feast-and-famine cycles may have altered metabolic profiles in Asian populations such that fat distribution is more concentrated in the abdominal region. Historically, the European region appears to undergo fewer famine cycles than Asian regions of the world. This can be attributed to historical patterns of development: efficient trading and distribution systems, centrality in both land and sea commerce, and agricultural diversification through importation of New World crops via the Columbian Exchange [34]. Conversely, the Indian subcontinent endures frequent monsoon seasons where agricultural yield and food availability depend on seasonal fluctuations. Repeating seasonal changes and resultant periods of starvation may be accompanied by metabolic alterations in which the body attempts to compensate by altering its metabolic functioning - and possibly anthropomorphic fat distribution - in order to maximize scarce resources and promote survival [71]. The feast-and-famine phenomenon may explain why the increase in diabetes within the population in the past decades has been lower for Europeans and higher for regions undergoing rapid economic change and modernization [34]. Though the initial proposition analyzed an increased incidence of diabetes within the population, we include it in light of the common relationship between increased incidence of diabetes and visceral fat deposition within the Asian population. Being traditionally less exposed to famine conditions, increased caloric, sugar, and fat consumption (accompanying trends in globalization) results in a less marked increase in diabetes in European populations than in historically famine-exposed Asian populations in which once advantageous phenotypes are unable to adapt to sudden, dramatic dietary changes (accompanying trends in rapid economic development).
Therefore, we see how in both the short-term (thrifty phenotype and epigenotype changes) and long-term (inherited metabolic changes endemic to historically famine-exposed regions) environmental influences may contribute to modified metabolic function, with one significant result being altered abdominal and visceral fat distribution patterns in Asian population.

\section{MALAYSIA: AN ILLUSTRATIVE EXAMPLE OF THE INTRICATE RELATIONSHIP BETWEEN GENETICS AND THE ENVIRONMENT}

Among East and Southeast Asian countries, Malaysia has the highest rate of overweight and obese individuals (Fig. 1) [72,73]. Inspection of Fig. 1 reinforces this statement: from 1975 to 2015, the prevalence of overweight or obesity in Malaysia increased by an astonishing 30.3\%. As of 2019, 64\% of males and $65 \%$ of females were classified as overweight [72]. This stands in stark contrast to India, China, and Vietnam, where the prevalence of overweight or obesity increased by $13.8 \%, 23.8 \%$, and $12.9 \%$, respectively. This alarming trend has been accompanied by increases in diabetes, hypercholesterolemia, hypertension, and cardiovascular diseases [72,74]. Here, we highlight how the increased incidence of overweight and obesity in Malaysia can be analyzed from both a genetic and environmental standpoint.

\section{Environment}

Like other developing countries, industrialization brings increased exposure to Western norms, most significantly Western diets, which include highly processed meats, fast food, sugars (notably sweetened carbonated beverages), and fats [72,75]. However, compared to other Asian countries who have experienced comparable rates of industrialization and urbanization (China, Thailand, etc.), Malaysia demonstrates the highest levels of overweight and obesity in the population, as well as the most rapid increase in overweight and obese individuals. Compared to Vietnam, which has one of the lowest rates of overweight and obesity in Asia, Malaysia has exhibited a greater increase in gross domestic product (GDP) per capita from 1950 to 2016 [76]. The percentage of the population living in urban areas has increased by $54.48 \%$, from $20.36 \%$ in 1950 to $74.84 \%$ in 2016. This stands in stark contrast to Vietnam's increase of $22.87 \%$, from $11.64 \%$ in 1950 to $34.51 \%$ in 2016 [77]. While the majority of Vietnam's population remains largely rural, most of the Malaysian population resides in urban areas, which pro- 
vides increased chance for exposure to Western cuisine and culture and a movement away from traditional foodstuffs. Poor urban living conditions and industrial-sector employment may be accompanied by decreased access to traditionally farm-grown food options and increased accessibility to fast food, work-related stress, and stress caused by adjustment to city life and substandard living conditions (pollution, crowded housing, etc.). Socioeconomic status has been shown to be a noteworthy indicator of obesity risk, as economically disadvantaged persons often suffer food insecurity and lack access to healthier, albeit more expensive, food options [74].

A study by Koo et al. [78] of young adult university students in Malaysia provides insight into the need for educational food diversification programs among the younger generation. Students often resort to the most available and affordable food options, namely fast food and prepackaged snacks, which is accompanied by lower consumption of fruits and vegetables. Though caloric intake is met - and many times exceeded - diets are oftentimes lacking in micronutrients and fiber and abundant in processed carbohydrates, sugars, and fats. These dietary habits come at a time of increased stress in transitioning from childhood to adulthood and adjusting to university life; such unmoderated diet patterns accrued early in life often persist into adulthood [78]. In addition to the ongoing nutritional transition taking place in Malaysia, the country exhibits an increasingly aging population, as with many other countries undergoing rapid industrialization. Elderly individuals tend to be less active and therefore more susceptible to increased fat accumulation and obesity [79].

\section{Genetics}

Specific genes involved in sugar and fat metabolism may predispose some individuals to metabolic syndrome and obesity. A study of the $A D R B 2$ rs 1042713 allele demonstrated an increased risk of insulin resistance and higher cholesterol levels in Malaysian carriers of the G allele [80]. These effects were observed only in obese individuals, which may suggest possible gene-diet interaction. Preliminary data indicated potential metabolic and glycemic benefits of incorporating polyunsaturated fats into the diets of individuals carrying the $\mathrm{G}$ allele, suggesting dietary modifications and educational awareness may help in stemming the current health crisis despite possible genetic predispositions.

A deeper exploration into Malaysian genetic ancestry may provide insight into current epidemiological trends, specifically shared ancestry between ancestral Malaysian and Polynesian people. An analysis of Polynesian DNA indicated possible
Southeast Asian origins, suggesting that ancestral Polynesian persons migrated via boat from the Asian continent to the islands of Oceania [81].

As of 2016, the top 10 countries ranked by obesity prevalence are all located in Oceania. Nauru ranks the highest in the world, with $61.00 \%$ of the population classified as obese, followed by the Cook Islands, Palau, Marshall Islands, and Tuvalu at $55.90 \%, 55.30 \%, 52.90 \%$, and $51.60 \%$, respectively [82]. In combination with its high obesity rate, Nauru has the world's highest rate of type 2 diabetes at $40 \%$ of the population [83]. A history of colonialization, industrialization, and the country's subsequent nutritional transition (phosphate mining and the destruction of arable land, followed by a decline in consumption of fresh fruits and root vegetables and increased importation of processed foodstuffs, especially fatty meats) contributed to the country's rapid rise in obesity and related metabolic syndromes. However, considering equally rapid and exhaustive industrialization trends in other nations, one must ask whether there is a genetic component that predisposes inhabitants of the islands of Oceania to patterns of increased fat accumulation so much so that the region contains the countries with the world's highest rates of obesity.

Malaysia and Polynesia's industrial and nutritional transition occurred relatively recently compared to the timescale required for evolutionary change. We suggest that gene-diet interactions facilitated these astonishing increases in obesity (Fig. 2). Polynesian settlers may have common ancestry with ancestral Malay people, bringing with them their shared genetic lineage, which may include "nutritionally thrifty" genes brought about by feastand-famine cycles endemic to the region. Genetic material programmed for "nutritional thrift" during ancestral feast-and-famine cycles perform disadvantageously when exposed to environments of nutritional abundance high in processed sugars and fats. If such a shared genomic ancestry and its accompanying metabolic predispositions do exist, this may partially explain Malaysia's anomalous increased rates of obesity compared to its neighboring Asian countries.

As such, the epidemiological situation in Malaysia demonstrates the intricate interplay between shared ancestral lineage, genetic predisposition, and environmental influences (urbanization, physical activity, diet, etc.) on fat storage and its consequential health hazards.

\section{CONCLUSIONS}

This review discussed potential ethnic differences in fat storage/ 
metabolism and how such differences may contribute to higher incidences of metabolic disease such as type 2 diabetes among Asians. Currently available data suggest that there are clear differences in fat distribution along ethnic lines. In general, Asians tend to accumulate higher visceral fat over subcutaneous fat when compared to Africans, Hispanics, and Caucasians. The current literature suggests that these variations are largely due to intrinsic genetic differences and physical features restricting fat accumulation in certain areas. However, biological genetic differences are increasingly modified and influenced by the surrounding environment (diet, physical activity, lifestyle, stress, etc.) over thousands of years.

It is well established that East Asian females tend to accumulate less fat in their hips than their Hispanic and African counterparts. There is also regional and ethnic heterogeneity in fat storage within the Asian population, such as different fat distribution patterns among South Asians and East Asians. It is unclear whether genetic differences in fat accumulation between ethnic groups evolved over an extended period, or if metabolic and adipose storage differences had any selective advantage. Further, one may wonder whether consumption of certain types of food (regional staples such as rice) and/or its abundance/scarcity may have contributed to epigenetic changes and altered fat storage in the human population. Culture (individualistic vs. collectivistic) and its influence on day-to-day behaviors (dietary staples, exercise frequency, sedentary tendencies, familial over individual prioritizations, etc.) may play a key role in Asian fat storage patterns. Understanding and targeting cultural propensities may prove valuable in providing ways to encourage healthy exercise habits within the Asian population, especially in the older generation. Hence, future research may focus on ways in which collectivistic cultural values can be applied to develop healthier diets and exercise practices (group and family exercise and educational programs, for instance).

We propose a collective theory on fat storage in Asian populations through analyzing current obesity and overweight trends in Malaysia, which we call the Malaysian gene-environment multiplier hypothesis. Ancestral feast-and-famine cycles endemic to certain Asian regions favored genes with selective advantages ("nutritional thrift") that are then passed down in members of shared genomic heritage. These gene-diet interactions are negatively exploited when inheritors of nutritionally thrifty genes are exposed to modern-day environmental pressures (highly processed diets, sedentary lifestyles, urbanization, work-related and cultural stressors, etc.). The action of nutritionally thrifty genes upon encountering modern-day environ- mental pressures may predispose or contribute to metabolic syndromes that are characteristic of certain ethnic groups. This gene-diet interaction is one built up over thousands of years, but effectively "multiplied" over a short period of time, as in the case of Malaysia and Polynesia's rapid increase in obesity and overweight following accelerated development and exposure to modern-day environmental pressures. Key aspects of the Malaysian gene-environment multiplier hypothesis may be similarly applied to other Asian countries; however, further research is needed to determine the degree to which other Asian countries exhibit these trends, as well as whether such trends are observable on the same scale as seen in Malaysia.

It appears that genetics sets a foundational baseline for fat storage, distribution, and metabolism, but environmental factors and lifestyle changes are becoming increasingly influential and critical in the modern-day world. Future research should focus on fat metabolism and storage in different ethnic backgrounds and the genetic, environmental, cultural, and behavioral components that influence such patterns. Identifying key ethnic differences in fat metabolism may provide future advantages in personalized treatments of metabolic syndromes in Asian populations.

The information presented in this review is limited to current published knowledge obtained from database searches; no original research project was undertaken. The discussion of Asian abdominal adiposity predominantly focused on the adult age group. There is also a paucity of data with regard to genetic determinants influencing obesity. While some Asian countries such as Japan and Korea are highly homogenous, others such as India and Malaysia exhibit significant diversity in their ethnic composition. Although ethnic differences among Asians were considered, variations in ethnic composition, as well as regional variations in abdominal adiposity, within the Asian countries mentioned were not considered. Other socioeconomic factors were not considered, but the prevalence of abdominal obesity appeared higher in urban populations than in rural populations.

\section{CONFLICTS OF INTEREST}

No potential conflict of interest relevant to this article was reported.

\section{ACKNOWLEDGMENTS}

This work was supported in part, by National Institutes of Health Grants R01 DK098240-01 and funding from UCF Col- 
lege of Medicine. We would like to thank several colleagues, Drs Naresh Bal, Meghna Pant, Shang-Shang Gao, Wencai Zhang, and Hung Nguyen, for feedback and critical reading of the manuscript. We thank Doque Williams for the artwork included in the manuscript.

\section{ORCID}

Rachel Williams https://orcid.org/0000-0002-0539-3857

Muthu Periasamy https://orcid.org/0000-0001-8834-5975

\section{REFERENCES}

1. World Health Organization. Obesity and Overweight [Internet]. Geneva: World Health Organization; 2020 [cited 2020 Nov 9]. Available from: https:/www.who.int/en/news-room/ fact-sheets/detail/obesity-and-overweight.

2. Flatt JP. Use and storage of carbohydrate and fat. Am J Clin Nutr 1995;61(4 Suppl):952S-9S.

3. Ibrahim MM. Subcutaneous and visceral adipose tissue: structural and functional differences. Obes Rev 2010;11:11-8.

4. Wang S, Liu Y, Li F, Jia H, Liu L, Xue F. A novel quantitative body shape score for detecting association between obesity and hypertension in China. BMC Public Health 2015;15:7.

5. Mongraw-Chaffin M, Golden SH, Allison MA, Ding J, Ouyang $\mathrm{P}$, Schreiner PJ, et al. The sex and race specific relationship between anthropometry and body fat composition determined from computed tomography: evidence from the multiethnic study of atherosclerosis. PLoS One 2015;10:e0139559.

6. Bray GA, Bouchard C. Handbook of obesity. 3rd ed. Boca Raton: CRC Press; 2014. Chapter 7, Gender, ethnic, and geographic variation in adiposity; p. 75-86.

7. Lim U, Ernst T, Buchthal SD, Latch M, Albright CL, Wilkens LR, et al. Asian women have greater abdominal and visceral adiposity than Caucasian women with similar body mass index. Nutr Diabetes 2011;1:e6.

8. Liu A, Byrne NM, Kagawa M, Ma G, Kijboonchoo K, Nasreddine L, et al. Ethnic differences in body fat distribution among Asian pre-pubertal children: a cross-sectional multicenter study. BMC Public Health 2011;11:500.

9. Kadowaki S, Miura K, Kadowaki T, Fujiyoshi A, El-Saed A, Masaki $\mathrm{KH}$, et al. International comparison of abdominal fat distribution among four populations: the ERA-JUMP study. Metab Syndr Relat Disord 2018;16:166-73.

10. Garg SK, Lin F, Kandula N, Ding J, Carr J, Allison M, et al. Ectopic fat depots and coronary artery calcium in South
Asians compared with other racial/ethnic groups. J Am Heart Assoc 2016;5:e004257.

11. Stults-Kolehmainen MA, Stanforth PR, Bartholomew JB, Lu T, Abolt CJ, Sinha R. DXA estimates of fat in abdominal, trunk and hip regions varies by ethnicity in men. Nutr Diabetes 2013;3:e64.

12. Park YW, Allison DB, Heymsfield SB, Gallagher D. Larger amounts of visceral adipose tissue in Asian Americans. Obes Res 2001;9:381-7.

13. Wulan SN, Westerterp KR, Plasqui G. Ethnic differences in body composition and the associated metabolic profile: a comparative study between Asians and Caucasians. Maturitas 2010;65:315-9.

14. Slentz CA, Aiken LB, Houmard JA, Bales CW, Johnson JL, Tanner CJ, et al. Inactivity, exercise, and visceral fat. STRRIDE: a randomized, controlled study of exercise intensity and amount. J Appl Physiol (1985) 2005;99:1613-8.

15. Bi X, Loo YT, Henry CJ. Android fat as a determinant of metabolic syndrome: sex differences. Nutrition 2019;57:127-32.

16. Valsamakis G, Chetty R, Anwar A, Banerjee AK, Barnett A, Kumar S. Association of simple anthropometric measures of obesity with visceral fat and the metabolic syndrome in male Caucasian and Indo-Asian subjects. Diabet Med 2004;21: 1339-45.

17. Saeedi P, Petersohn I, Salpea P, Malanda B, Karuranga S, Unwin N, et al. Global and regional diabetes prevalence estimates for 2019 and projections for 2030 and 2045: results from the International Diabetes Federation Diabetes Atlas, 9th edition. Diabetes Res Clin Pract 2019;157:107843.

18. Yajnik CS, Yudkin JS. The Y-Y paradox. Lancet 2004;363: 163.

19. Lotta LA, Wittemans LB, Zuber V, Stewart ID, Sharp SJ, Luan J, et al. Association of genetic variants related to gluteofemoral vs abdominal fat distribution with type 2 diabetes, coronary disease, and cardiovascular risk factors. JAMA 2018;320:2553-63.

20. Heid IM, Jackson AU, Randall JC, Winkler TW, Qi L, Steinthorsdottir V, et al. Meta-analysis identifies 13 new loci associated with waist-hip ratio and reveals sexual dimorphism in the genetic basis of fat distribution. Nat Genet 2010; 42:949-60.

21. Shungin D, Winkler TW, Croteau-Chonka DC, Ferreira T, Locke AE, Magi R, et al. New genetic loci link adipose and insulin biology to body fat distribution. Nature 2015;518: 187-96.

22. Lin X, Qi Q, Zheng Y, Huang T, Lathrop M, Zelenika D, et 
al. Neuropeptide Y genotype, central obesity, and abdominal fat distribution: the POUNDS LOST trial. Am J Clin Nutr 2015;102:514-9.

23. Heymsfield SB, Peterson CM, Thomas DM, Heo M, Schuna JM Jr. Why are there race/ethnic differences in adult body mass index-adiposity relationships?: a quantitative critical review. Obes Rev 2016;17:262-75.

24. Lim U, Monroe KR, Buchthal S, Fan B, Cheng I, Kristal $\mathrm{BS}$, et al. Propensity for intra-abdominal and hepatic adiposity varies among ethnic groups. Gastroenterology 2019;156: 966-75.

25. Pulit SL, Karaderi T, Lindgren CM. Sexual dimorphisms in genetic loci linked to body fat distribution. Biosci Rep 2017; 37:BSR20160184.

26. Azuma K, Kadowaki T, Cetinel C, Kadota A, El-Saed A, Kadowaki S, et al. Higher liver fat content among Japanese in Japan compared with non-Hispanic whites in the United States. Metabolism 2009;58:1200-7.

27. Cook WK, Tseng W, Bautista R, John I. Ethnicity, socioeconomic status, and overweight in Asian American adolescents. Prev Med Rep 2016;4:233-7.

28. Paley C, Hull H, Ji Y, Toro-Ramos T, Thornton J, Bauer J, et al. Body fat differences by self-reported race/ethnicity in healthy term newborns. Pediatr Obes 2016;11:361-8.

29. He Q, Horlick M, Thornton J, Wang J, Pierson RN Jr, Hesh$\mathrm{ka} \mathrm{S}$, et al. Sex and race differences in fat distribution among Asian, African-American, and Caucasian prepubertal children. J Clin Endocrinol Metab 2002;87:2164-70.

30. Freedman DS, Wang J, Thornton JC, Mei Z, Pierson RN Jr, Dietz WH, et al. Racial/ethnic differences in body fatness among children and adolescents. Obesity (Silver Spring) 2008;16:1105-11.

31. Shaw NJ, Crabtree NJ, Kibirige MS, Fordham JN. Ethnic and gender differences in body fat in British schoolchildren as measured by DXA. Arch Dis Child 2007;92:872-5.

32. Fox A, Feng W, Asal V. What is driving global obesity trends?: globalization or "modernization"? Global Health 2019;15:32.

33. Jackson P, Spiess WEL, Sultana F. eating, drinking: surviving the international year of global understanding-IYGU. Cham: Springer International Publishing; 2016. Chapter, The Nutrition Transition in Developing Asia: Dietary Change, Drivers and Health Impacts; p. 83-8.

34. Siddiqui K, Joy SS, Nawaz SS. Impact of early life or intrauterine factors and socio-economic interaction on diabetes: an evidence on thrifty hypothesis. J Lifestyle Med 2019;9:
92-101.

35. Ferreira SR, Lerario DD, Gimeno SG, Sanudo A, Franco LJ; Japanese-Brazilian Diabetes Study Group. Obesity and central adiposity in Japanese immigrants: role of the Western dietary pattern. J Epidemiol 2002;12:431-8.

36. Shimabukuro M, Higa M, Kinjo R, Yamakawa K, Tanaka H, Kozuka C, et al. Effects of the brown rice diet on visceral obesity and endothelial function: the BRAVO study. Br J Nutr 2014;111:310-20.

37. Krittanawong C, Tunhasiriwet A, Zhang H, Prokop LJ, Chirapongsathorn $\mathrm{S}$, Sun $\mathrm{T}$, et al. Is white rice consumption a risk for metabolic and cardiovascular outcomes?: a systematic review and meta-analysis. Heart Asia 2017;9:e010909.

38. Seo MH, Lee WY, Kim SS, Kang JH, Kang JH, Kim KK, et al. 2018 Korean Society for the Study of Obesity guideline for the management of obesity in Korea. J Obes Metab Syndr 2019;28:40-5.

39. Shin JH, Jung S, Kim SA, Kang MS, Kim MS, Joung H, et al. Differential effects of typical Korean versus Americanstyle diets on gut microbial composition and metabolic profile in healthy overweight Koreans: a randomized crossover trial. Nutrients 2019;11:2450.

40. Kyrou I, Tsigos C. Stress hormones: physiological stress and regulation of metabolism. Curr Opin Pharmacol 2009;9: 787-93.

41. Drapeau V, Therrien F, Richard D, Tremblay A. Is visceral obesity a physiological adaptation to stress? Panminerva Med 2003;45:189-95.

42. Chrousos GP. The role of stress and the hypothalamic-pituitary-adrenal axis in the pathogenesis of the metabolic syndrome: neuro-endocrine and target tissue-related causes. Int J Obes Relat Metab Disord 2000;24 Suppl 2:S50-5.

43. Vinet A, Obert P, Courteix D, Chapier R, Lesourd B, Verney J, et al. Different modalities of exercise improve macrovascular function but not microvascular function in metabolic syndrome: the RESOLVE randomized trial. Int J Cardiol 2018;267:165-70.

44. Irving BA, Davis CK, Brock DW, Weltman JY, Swift D, Barrett EJ, et al. Effect of exercise training intensity on abdominal visceral fat and body composition. Med Sci Sports Exerc 2008;40:1863-72.

45. Hunter GR, Brock DW, Byrne NM, Chandler-Laney PC, Del Corral P, Gower BA. Exercise training prevents regain of visceral fat for 1 year following weight loss. Obesity (Silver Spring) 2010;18:690-5.

46. Rimmele U, Zellweger BC, Marti B, Seiler R, Mohiyeddini 
C, Ehlert U, et al. Trained men show lower cortisol, heart rate and psychological responses to psychosocial stress compared with untrained men. Psychoneuroendocrinology 2007;32:627-35.

47. Milani RV, Lavie CJ. Reducing psychosocial stress: a novel mechanism of improving survival from exercise training. Am J Med 2009;122:931-8.

48. Mikkelsen K, Stojanovska L, Polenakovic M, Bosevski M, Apostolopoulos V. Exercise and mental health. Maturitas 2017;106:48-56.

49. Kennedy G, Hardman RJ, Macpherson H, Scholey AB, Pipingas A. How does exercise reduce the rate of age-associated cognitive decline?: a review of potential mechanisms. J Alzheimers Dis 2017;55:1-18.

50. Williams ED, Stamatakis E, Chandola T, Hamer M. Assessment of physical activity levels in South Asians in the UK: findings from the Health Survey for England. J Epidemiol Community Health 2011;65:517-21.

51. Owen CG, Nightingale CM, Rudnicka AR, Cook DG, Ekelund U, Whincup PH. Ethnic and gender differences in physical activity levels among 9-10-year-old children of white European, South Asian and African-Caribbean origin: the Child Heart Health Study in England (CHASE Study). Int J Epidemiol 2009;38:1082-93.

52. Kandula NR, Lauderdale DS. Leisure time, non-leisure time, and occupational physical activity in Asian Americans. Ann Epidemiol 2005;15:257-65.

53. Misra A, Nigam P, Hills AP, Chadha DS, Sharma V, Deepak $\mathrm{KK}$, et al. Consensus physical activity guidelines for Asian Indians. Diabetes Technol Ther 2012;14:83-98.

54. Babakus WS, Thompson JL. Physical activity among South Asian women: a systematic, mixed-methods review. Int J Behav Nutr Phys Act 2012;9:150.

55. Bhatnagar P, Shaw A, Foster C. Generational differences in the physical activity of UK South Asians: a systematic review. Int J Behav Nutr Phys Act 2015;12:96.

56. Horne M, Tierney S. What are the barriers and facilitators to exercise and physical activity uptake and adherence among South Asian older adults: a systematic review of qualitative studies. Prev Med 2012;55:276-84.

57. Waidyatilaka I, Lanerolle P, Wickremasinghe R, Atukorala S, Somasundaram N, de Silva A. Sedentary behaviour and physical activity in South Asian women: time to review current recommendations? PLoS One 2013;8:e58328.

58. Smith L, Aggio D, Hamer M. Longitudinal patterns in objective physical activity and sedentary time in a multi-ethnic sample of children from the UK. Pediatr Obes 2018;13:120-6.

59. Smith L, Lopez Sanchez GF, Diaz Suarez A, Stubbs B, Dowling M, Scruton A, et al. Barriers and facilitators of physical activity in children of a South Asian ethnicity. Sustainability 2018;10:761.

60. Carver A, Akram M, Barnett A, Mellecker R, Cerin E. Socioeconomic status and physical activity among mothers of young children in an Asian city: the mediating role of household activities and domestic help. Int J Environ Res Public Health 2020;17:2498.

61. Yan Z, Cardinal BJ. Association of Asian/Asian-American immigrants physical activity behavior and length of residence in the U.S. J Behav Health 2019;8:66-70.

62. Neel JV. Diabetes mellitus: a "thrifty" genotype rendered detrimental by "progress"? Am J Hum Genet 1962;14:35362.

63. Speakman JR. Thrifty genes for obesity, an attractive but flawed idea, and an alternative perspective: the 'drifty gene' hypothesis. Int J Obes (Lond) 2008;32:1611-7.

64. Hales CN, Barker DJ. Type 2 (non-insulin-dependent) diabetes mellitus: the thrifty phenotype hypothesis: 1992. Int J Epidemiol 2013;42:1215-22.

65. Stoger R. The thrifty epigenotype: an acquired and heritable predisposition for obesity and diabetes? Bioessays 2008;30: 156-66.

66. Ozanne SE, Constancia M. Mechanisms of disease: the developmental origins of disease and the role of the epigenotype. Nat Clin Pract Endocrinol Metab 2007;3:539-46.

67. Li Y, He Y, Qi L, Jaddoe VW, Feskens EJ, Yang X, et al. Exposure to the Chinese famine in early life and the risk of hyperglycemia and type 2 diabetes in adulthood. Diabetes 2010;59:2400-6.

68. De Rooij SR, Painter RC, Phillips DI, Osmond C, Michels RP, Godsland IF, et al. Impaired insulin secretion after prenatal exposure to the Dutch famine. Diabetes Care 2006;29: 1897-901.

69. Tobi EW, Goeman JJ, Monajemi R, Gu H, Putter H, Zhang Y, et al. DNA methylation signatures link prenatal famine exposure to growth and metabolism. Nat Commun 2014;5: 5592.

70. Whitelaw NC, Whitelaw E. How lifetimes shape epigenotype within and across generations. Hum Mol Genet 2006; 15 Spec No 2:R131-7.

71. Wells JC. Commentary: why are South Asians susceptible to central obesity?: the El Nino hypothesis. Int J Epidemiol 2007;36:226-7. 
72. Kaur N. Malaysia and WHO call for more investment in primary health care the 21st century Kuala Lumpur, Malaysia [Internet]. Geneva: World Health Organization; 2019 [cited 2020 Nov 9]. Available from: https:/www.who.int/malaysia/ news/detail/08-04-2019-malaysia-and-who-call-for-moreinvestment-in-primary-health-care-the-21st-century.

73. Ritchie H, Roser M. Obesity [Internet]. ourworldindata; 2018 [cited 2020 Nov 9]. Available from: https://ourworldindata. org/obesity.

74. Law LS, Sulaiman N, Gan WY, Adznam SN, Mohd Taib MN. Predictors of overweight and obesity and its consequences among Senoi Orang Asli (indigenous people) women in Perak, Malaysia. Int J Environ Res Public Health 2020; $17: 2354$.

75. Noor MI. The nutrition and health transition in Malaysia. Public Health Nutr 2002;5(1A):191-5.

76. Roser M. Economic growth: our world in data [Internet]. ourworldindata; 2013 [cited 2020 Nov 9]. Available from: https://ourworldindata.org/economic-growth.

77. Ritchie H, Roser M. Urbanization [Internet]. ourworldindata; 2018 [cited 2020 Nov 9]. Available from: https://ourworldindata.org/urbanization.

78. Koo HC, Hadirah Z, Airina A, Nurul Alifatul Amrina R, Faziela N. Effect of nutrient intakes on anthropometric profiles among university students from a selected private University in Klang Valley, Malaysia. Afr Health Sci 2019;19:
2243-51.

79. Chan YY, Sooryanarayana R, Mohamad Kasim N, Lim KK, Cheong SM, Kee CC, et al. Prevalence and correlates of physical inactivity among older adults in Malaysia: findings from the National Health and Morbidity Survey (NHMS) 2015. Arch Gerontol Geriatr 2019;81:74-83.

80. Mitra SR, Tan PY, Amini F. Association of ADRB2 rs1042713 with obesity and obesity-related phenotypes and its interaction with dietary fat in modulating glycaemic indices in Malaysian adults. J Nutr Metab 2019;2019:8718795.

81. Kayser M, Lao O, Saar K, Brauer S, Wang X, Nurnberg P, et al. Genome-wide analysis indicates more Asian than Melanesian ancestry of Polynesians. Am J Hum Genet 2008;82: 194-8.

82. Central Intelligence Agency. The world factbook: obesity adult prevalence rate [Internet]. CIA; 2016 [cited 2020 Nov 9]. Available from: https://www.cia.gov/library/publications/the-world-factbook/rankorder/2228rank.html.

83. The astonishing story of Nauru, the tiny island nation with the world's highest rates of type 2 diabetes [Internet]. Diabetes.co.uk; 2019 [cited 2020 Nov 9]. Available from: https:// www.diabetes.co.uk/in-depth/i-have-seen-so-many-funeralsfor-such-a-small-island-the-astonishing-story-of-nauru-thetiny-island-nation-with-the-worlds-highest-rates-of-type2-diabetes. 